\title{
PD-1 and PD-L1 Expression in Indian Women with Breast Cancer
}

(D) Kishan R. Bharadwaj ${ }^{1}$, (1) Kuheli Dasgupta ${ }^{2}$, (1) Suma Mysore Narayana ${ }^{3}$, (1) C. Ramachandra ${ }^{4}$, (1) Suresh M.C. Babu ${ }^{5}$, (D) Annapoorni Rangarajan², (1) Rekha V. Kumar ${ }^{3}$

${ }^{1}$ IISc Mathematics Initiative, Indian Institute of Science (IISc), Bangalore, India

${ }^{2}$ Department of Molecular Reproduction Development and Genetics, Indian Institute of Science (IISc), Bangalore, India

${ }^{3}$ Department of Pathology, Kidwai Memorial Institute of Oncology (KMIO), Bangalore, India

${ }^{4}$ Department of Surgical Oncology, Kidwai Memorial Institute of Oncology (KMIO), Bangalore, India

${ }^{5}$ Department of Medical Oncology, Kidwai Memorial Institute of Oncology (KMIO), Bangalore, India

\section{ABSTRACT}

Objective: The interaction between programmed cell death protein 1 (PD-1) on activated T-lymphocytes and programmed death-ligand 1 (PD-L1) on tumor cells or antigen-presenting cells sends immunosuppressive signals leading to the escape of tumor cells from the host anti-tumor immune response. Inhibiting this interaction with antibodies against PD-1 or PD-L1 is emerging as a valuable therapeutic strategy. However, tissue distribution patterns for PD-L1 and PD-1 in breast cancer patients from India are not reported, yet many clinical trials are underway. In this study the expression of PD-1 and PDL1 in breast cancer patient samples from India was characterized.

Materials and Methods: The study included 392 cases of operated breast cancer (2012-2017) from a tertiary cancer care center in Bangalore, Karnataka, India. Paraffin blocks were retrievable and receptor status was known. Immunohistochemistry (IHC) was performed using anti-PD-L1 and anti-PD-1 antibodies. RNA was isolated from 76 fresh tumors and nine adjacent normal tissues (2019). PD-L1 transcript levels were measured by RT-qPCR using Hypoxanthine-guanine phosphoribosyl transferase (HPRT) as a reference gene.

Results: Based on IHC, PD-1 expression within tumor-infiltrating immune cells (TIICs) was observed in 55/385 cases (14\%) across all breast cancer types. In triple-negative breast cancer (TNBC), 21/132 cases (16\%) showed PD-1 staining in TIICs. The overall expression of PD-L1 in breast tumor cells across all breast cancer subtypes and TIICs was 11\% (41/378) and 39\% (151/385), respectively. A relatively higher proportion of TNBC cases had PD-L1 expression in tumor cells (17/132 cases, 13\%) and immune cells (68/132 cases, 52\%). We also detected PD-L1 transcript expression by qRT-PCR in freshly isolated tumor samples.
\end{abstract}

Conclusion: These findings show that around 52\% (68/132) of the TNBC cases express PD-L1 in TIICs. Hence, anti-PD-1/PD-L1 therapy alone or combined with chemotherapy may be a promising treatment for TNBC in Indian patients.

Keywords: Triple-negative breast cancer, programmed cell death protein 1 (PD-1), programmed death-ligand 1 (PD-L1), immunohistochemistry

Cite this article as: Bharadwaj KR, Dasgupta K, Narayana SM, Ramachandra C, Babu SMC, Rangarajan A, Kumar RV. PD-1 and PD-L1 Expression in Indian Women with Breast Cancer. Eur J Breast Health 2022; 18(1): 21-29

\section{Key Points}

- $\quad$ PD-1 and PD-L1 expression was studied for the first time in breast cancer patient samples from India.

- $33 \%$ of the breast cancer cases were triple negative breast cancer (TNBC).

- $64 \%$ of the TNBC cases showed immune response.

- $\quad$ About $13 \%$ of the TNBC cases had tumor cells expressing PD-L1.

- $\quad$ Around $52 \%$ of TNBC cases had tumor infiltrating immune cells expressing PD-L1. 


\section{Introduction}

Breast cancer is the leading cause of death due to cancer among women in the world (1). In India, $14 \%$ of the cancer incidence and $11 \%$ of cancer mortalities are due to breast cancer (1). Breast cancer is classified into six subtypes, based on gene expression microarray analysis, known as intrinsic subtype classification. The six subtypes are luminal A, luminal B, human epidermal growth factor receptor 2 (HER2), basal-like, normal-like and Claudin-low breast cancer (2). The St. Gallen expert consensus on the primary therapy of breast cancer has released a surrogate classification for breast cancer subtypes to guide adjuvant treatment decisions. These surrogates were defined to distinguish luminal A-like breast cancer from luminal B-like, HER$2 /$ neu, and triple-negative disease, using a combination of estrogen receptor (ER), progesterone receptor (PR), Ki-67\%, and HER-2/ neu immunohistochemical (IHC) profiles, without a requirement for molecular diagnosis (3).

An effective immune system can identify and eliminate cancerous cells. Innate immune cells produce pro-inflammatory cytokines leading to an inflammatory response and tumor antigen presentation to adaptive immune cells, such as T-lymphocytes. Upon activation, T-lymphocytes eliminate the cancerous cells. Immune checkpoint pathways, such as the programmed cell death protein 1 /programmed death ligand 1 (PD-1/PD-L1) axis, regulate T-lymphocyte activity to prevent the destruction of 'self' cells (4).

PD-1 is a co-inhibitory receptor expressed predominantly by cytotoxic T lymphocytes (5). PD-1 interacts with its ligands, PD-L1 and PD$\mathrm{L} 2$, expressed by antigen-presenting cells. PD-L1 is generally expressed by tumor cells and macrophages, whereas PD-L2 is present mainly on dendritic cells $(5,6)$. On such an interaction, PD-1 signaling results in the attenuation of cytotoxic activity of $\mathrm{T}$ lymphocytes and promotes T-regulatory activity, leading to termination of host immune response $(5,7,8)$.

Recent studies have shown that cancer cells hijack this immune suppression mechanism by expressing PD-L1 on their surface and evade the host immune response $(5,9)$. Inhibitors of PD-1/PD-L1 immune checkpoints have been extensively explored in several cancers. The Food and Drug Administration (FDA) has approved anti-PD-1 and anti-PD-L1 immunotherapies for treating nine cancers, including melanoma, head and neck squamous cell carcinoma, urothelial and non-small cell lung carcinoma, among others (10).

Triple negative breast cancers (TNBCs) are more common in younger, premenopausal Indian women and are aggressive, with higher recurrence rates (11). Recent studies of gene expression of the breast cancer stroma have shown increased tumor-infiltrating immune cells and lymphocytic activity, especially in TNBCs (12). Based on the IMpassion130 clinical trial (NCT02425891), the FDA recently granted accelerated approval for Atezolizumab, a monoclonal antibody targeting PD-L1, plus chemotherapy (Abraxane; nabÒ-Paclitaxel) for the treatment of PD-L1-positive, unresectable, locally advanced, or metastatic TNBC (13).

Breast cancer was thought to be less immunogenic when compared to melanoma or non-small cell lung carcinoma. Some studies on PD-1 and PD-L1 levels in breast cancer across the world have reported contradicting correlations between PD-L1 expression and prognosis $(14,15)$. Furthermore, there is no data on PD-1 and PD-L1 expression the usefulness of anti-PD-L1 immunotherapy in Indian breast cancer patients by elucidating the expression patterns of PD-1 and PD-L1 in tumor and tumor-infiltrating immune cells in breast cancer patients in a regional cancer center in South India.

In clinical practice, immunohistochemistry (IHC) is used to evaluate PD-L1 expression in tumor cells and tumor-infiltrating immune cells (TIICs). In this study an IHC-based assessment of PD-1 and PD-L1 expression in a large-scale evaluation of a breast cancer patient cohort of Indian origin was performed. IHC staining depends on the affinity and avidity of the antibody used and the methodology. Hence, there is no universal cut-off to determine grade positivity (16). There is also interand intra-observer variation in the pathological scoring of cells by IHC. Therefore, the effectiveness of PD-L1 mRNA expression by quantitative real-time polymerase chain reaction (qRT-PCR) was also evaluated.

\section{Materials and Methods}

\section{Breast Cancer Subtype Scoring System}

Hormone receptor positivity $\left(H R_{+}\right)$was defined as either or both estrogen receptor (ER) positivity and progesterone receptor (PR) positivity. This was defined as a nuclear staining of any intensity of $\geq 1 \%$ of the tumor cells or an Allred Score of $\geq 3$.

Human epidermal growth factor receptor 2 positivity (HER-2+) was defined as complete and strong circumferential membranous staining of $>10 \%$ of tumor cells, scored 3+ with ER and PR being negative.

Triple-negative breast cancers (TNBC) were cases that were negative for all three markers: ER, PR and HER-2.

\section{Reagents Used for IHC and qRT-PCR}

Rabbit monoclonal PD-L1 antibody (ACI 3137C) and mouse monoclonal PD-1 antibody (ACI 3162C) were procured from Biocare (Biocare Inc., Concord, CA, USA) and used for IHC at a dilution of $1: 150$ and 1:80, respectively. PD-L1 and hypoxanthineguanine phosphoribosyl transferase (HPRT; Control) primers were designed in-house. Primer sequences are as follows: PDL1 (forward) - 5'-GGCATTTGCTGAACGCAT-3', PD-L1 (reverse) - 5- CAATTAGTGCAGCCAGGT-3', HPRT (forward) - 5'-TGCTCGAGATGTGATGAAGG-3' and HPRT (reverse) 5'- TCCCCTGTTGACTGGTCATT-3'. RNAlater (R0901) and TRIzol $^{\circledR}$ (T9424) were procured from Sigma Aldrich (Sigma Aldrich, St Louis, MO, USA). The reverse transcription kit (4368814) was obtained from Applied Biosystems, ThermoFisher, SYBR ${ }^{\oplus}$ green (BIO-98050) was procured from Bioline and ROX reference dye (RR390Q) was obtained from Takara Biosciences.

\section{Study Population}

Indian patients who had undergone modified radical mastectomy for invasive breast carcinoma between 2013 and 2017 were identified from the archives of the associated cancer hospital. Inclusion criteria were: patients with known ER/PR/HER2 status; and paraffinembedded tissue blocks were available. Exclusion criteria were: 1) a tumor type other than invasive carcinoma; 2) use of preoperative (neoadjuvant) chemotherapy; and 3) human immunodeficiency virus (HIV) seropositivity. Each resected specimen had undergone gross and histological examination by trained surgical pathologists. Paraffin-embedded blocks of retrospective cases were collected from the pathology department of the cancer hospital. All patient data were anonymized before study inclusion. 


\section{Ethical Approvals}

For prospective samples, patient consent was obtained in a written form before surgery. Both retrospective and prospective arms were approved by the Medical Ethics Committee (no: MEC/001, date: April 30, 2016). The tissue samples for RNA isolation were processed according to the Institute's human ethical clearance (IHEC) protocol of the research institute.

\section{Immunohistochemistry Procedure}

Cases of the three breast cancer subtypes (HR+, HER2+ and TNBC) from the years 2013-2017 were retrieved from the archives of the department of pathology. The hematoxylin and eosin (H\&E) stained sections were reviewed, and $4 \mu \mathrm{m}$ thick sections were cut from selected paraffin-embedded tissue blocks. The cut sections were mounted on silane-coated slides. IHC was performed using an automated immunostainer, Benchmark XT (Ventana Medical Systems, Inc., Tucson, Arizona, USA), according to the manufacturer's protocol. Sections were stained with diaminobenzidine and counterstained for the nucleus with hematoxylin for 30 seconds. Slides were then washed under slow-running tap water, air-dried, and mounted with DPX mounting agent. Bright-field images were taken using an Olympus IX71 (Olympus Corporation, Shinjuku-ku, Tokyo, Japan) inverted microscope using Image-Pro software.

\section{Evaluation of TIICs in H\&E Sections}

The H\&E stained sections that had been reviewed for tumor cell content earlier were semi-quantitatively examined to assess the extent of TIICs (Figures 1c-e). These were complete sections from excised specimens and did not include needle core biopsies or tissue microarray samples, given the heterogeneity of TIICs. There was no focus on hot spots. TIICs in tumor zones with crush artifact and necrosis were excluded. TIICs included all mononuclear cells; lymphocytes, macrophages and plasma cells. Granulocytes were excluded.

Only those TIICs within the borders of the invasive tumor were counted. Both intratumoral (immune cells in direct cell-to-cell contact with carcinoma cells with no intervening stroma) and stromal TIICs (immune cells dispersed in the stroma between the carcinoma cells and not directly in contact with carcinoma cells) were counted. Semiquantitative counting was done by an experienced pathologist. The percentage of TIICs was calculated as the area occupied by TIICs over the total intratumoral stromal area. These values were categorized into percentages: $0 \%, 1 \%-10 \%, 11 \%-50 \%$ and $>50 \%$.

\section{Assessment of PD-L1 and PD-1 immunostained sections}

After immunostaining, the sections were semi-quantitatively examined for positivity, as described below. The pathologist was blinded to the ER/PR/HER2 status of the cases when scoring.

Tumor cells were labeled positive for PD-L1 if staining at 200x magnification was present in greater than or equal to one percent of tumor cells $(\geq 1 \%)$, with partial or complete membrane staining, of any intensity. This method of scoring took into account the general definition of PD-L1-positive (PD-L1+) tumor cells (17). PD-L1

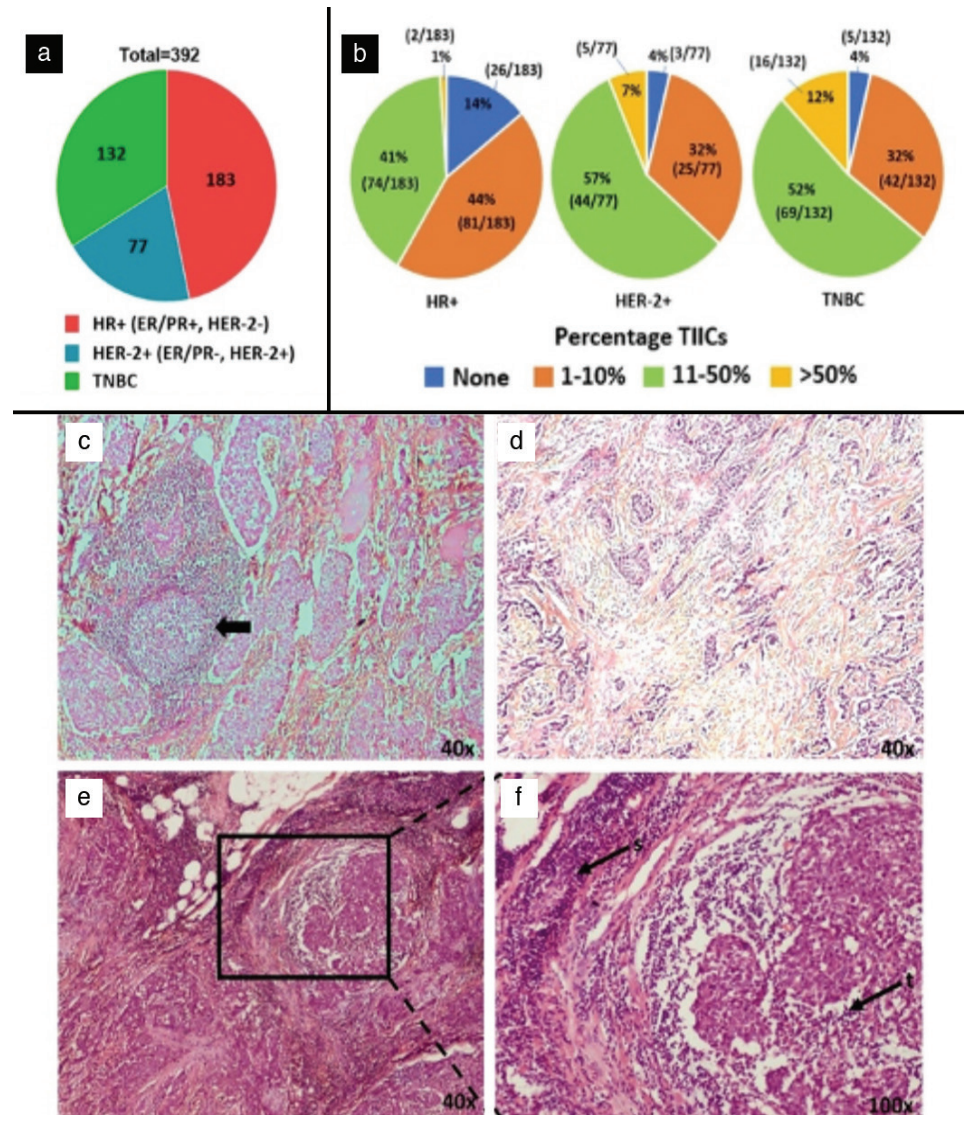

Figure 1. a) Pie chart showing the number of retrospective cases $(n=392)$ among various subtypes of breast cancer. $\mathbf{b})$ Pie charts showing tumor infiltrating immune cells (TIICS) across various breast cancer subtypes. The number of immune cells per field in tumor sections was divided into four categories: $0 \%$ cells, $1 \%-10 \%$ cells (mild), $11 \%-50 \%$ cells (moderate), $>50 \%$ cells (dense). c) TIICs as lymphoid follicles (arrow) with germinal centers. Representative images for d) mild TIICs (1\%-10\%), e) dense TIICs (>50\%). f) Magnified image of e. Immune cells infiltrating tumor ( $t)$ and stroma (s) 
positive tumor cells are those tumor cells that show partial or complete membranous staining for PD-L1 of any intensity. In practice, this would mean staining of $\geq 1 \%$ tumor cells. The magnification of 200x was chosen to assess PD-L1 staining in tumor cells and TIICs, as described by Salgado et al. (18), who assessed tumor-infiltrating lymphocytes (TILs) at the same magnification. Percentage categories were again defined as $0 \%,<5 \%, 5 \%-50 \%,>50 \%$, although this was later modified to $<10 \%$ and $\geq 10 \%$. The Tumor Proportion Score (TPS) was given using the following formula:

TPS $=($ Number of PD-L1 stained tumor cells/Total number of non-necrotic tumor cells) $\times 100$

TIICs were scored positive for PD-L1 if staining at 200x magnification was present in $\geq 1 \%$ of immune cells, either nuclear or cytoplasmic or both, of any intensity. Percentage categories were again defined: $0 \%,<5 \%, 5 \%-10 \%, 11 \%-50 \%,>50 \%$. The Mononuclear Immune Density Score (MIDS) was calculated using the following equation:

\section{MIDS $=($ Number of $P D-L 1$ positive immune cells/Total number} of non-necrotic tumor cells) $\times 100$

Similarly, TIICs were scored positive for PD-1 if staining at 200x magnification was present in $\geq 1 \%$ of immune cells, either membranous or cytoplasmic or both, of any intensity. The formula for MIDS was again used to calculate results, substituting the count of PD-1 cells for the count of PD-L1 cells used in the formula described above.

\section{Sample Collection and RNA Isolation}

Excision specimens were received in the Histopathology laboratory. Regions of the tumor were identified by the pathologist and at least 100 mg samples were taken from the tumor and adjacent grossly normal tissue at least $4 \mathrm{~cm}$ away from the tumor. Tissue samples were washed in $1 \mathrm{x}$ phosphate-buffered saline containing $1 \%$ penicillin and $1 \%$ streptomycin to remove surface contaminants and blood. The washed tissue was cut to approximately $1 \times 1 \times 0.2 \mathrm{~cm}^{3}$ and was transferred to a sterile $15 \mathrm{~mL}$ tube containing RNALater ${ }^{\circ}$ (Sigma Aldrich, St Louis, MO, USA) and allowed to stand at room temperature overnight. The tubes with samples were then stored frozen at $-20{ }^{\circ} \mathrm{C}$ until analysis.

Tissue samples were retrieved, thawed on ice, washed with Milli-Q water, and put in a $1.5 \mathrm{~mL}$ tube containing TRIzol $^{\oplus}$ reagent (Invitrogen, Thermo Fisher Scientific, Waltham, MA, USA). The tissue was homogenized using a handheld homogenizer and RNA was isolated according to TRIzol ${ }^{\odot}$ reagent's manufacturer's protocol.

\section{cDNA Preparation and qRT-PCR}

Two $\mu \mathrm{g}$ of the isolated RNA was used for cDNA synthesis using the reverse transcription kit (Applied Biosystems, Thermo Fisher Scientific, Waltham, MA, USA). cDNA samples were diluted 1:10 by taking $10 \mu \mathrm{L}$ of cDNA samples into $90 \mu \mathrm{L}$ of nuclease-free water. For qPCR reaction, one $\mu \mathrm{L}$ of the diluted sample was mixed with one $\mu \mathrm{L}$ of $1 \mathrm{X} \mathrm{SYBR}^{\oplus}$ Green Master mix (Bioline, Meridian Biosciences, Cincinnati, $\mathrm{OH}, \mathrm{USA}$ ) and $0.2 \mu \mathrm{L}$ of ROX passive reference dye at a final concentration of $500 \mathrm{nM}$ (Takara Biosciences, Shiga, Japan). Nuclease-free water was added to make up the final volume to 10 $\mu L$. Primer concentration was maintained at $10 \mu \mathrm{M}$ for the qPCR reactions. qPCR was performed in a real-time PCR thermocycler (Applied Biosystems, Thermo Fisher Scientific, Waltham, MA, USA) with 40 cycles of denaturation at $95^{\circ} \mathrm{C}$ for 30 seconds, annealing at extension at $72{ }^{\circ} \mathrm{C}$ for 10 minutes was given for re-annealing of the PCR products into double-stranded DNA. To ensure complete reannealing, the temperature was then lowered and held at $60{ }^{\circ} \mathrm{C}$ for 1 minute. A melting curve analysis was done by increasing temperature stepwise to $95^{\circ} \mathrm{C}$ using $1{ }^{\circ} \mathrm{C} /$ minute steps.

PD-L1 mRNA levels in the tumor and adjacent normal tissue were evaluated by normalizing the threshold cycle number $(\mathrm{Ct})$ of PD-L1 with the $\mathrm{Ct}$ of a housekeeping gene, HPRT. PD-L1 mRNA levels across tumor samples were analyzed by plotting patient code number versus $2^{(-\Delta \mathrm{Ct})}$, where $\Delta \mathrm{Ct}$ was calculated by subtracting $\mathrm{Ct}$ of PD-L1 from Ct of HPRT. The fold change in the PD-L1 mRNA in tumors, when compared to matched adjacent normal tissue, was estimated as $2^{(-\Delta \Delta \mathrm{C} t)}$, where $\Delta \Delta \mathrm{Ct}$ was calculated by subtracting $\Delta \mathrm{Ct}$ of tumor from $\Delta \mathrm{Ct}$ of adjacent normal tissue.

\section{Results}

\section{High Triple-Negative Breast Cancer Cases in India}

A total of 392 cases were retrieved from the archives of the department of pathology. The distribution of cases based on ER, PR and HER2 status is given in Figure 1a. The majority (48\%) of cases were HR+, $19 \%$ was HER $2+$ and $33 \%$ was TNBCs (Table 1). Studies from different parts of India have shown a similar trend. One study with a cohort of 5,436 patients had shown a similar trend with 48\% HR+, $15 \%$ HER $2+$ and $37 \%$ TNBC (19). Another study $(\mathrm{n}=123)$ from a North-Eastern state of India showed a trend of 40.6\% HR+, $17.9 \%$ HER $2+$ and 38.2\% TNBC while another study $(n=2,062)$ from the Western part of the country reported $44.6 \% \mathrm{HR}+, 11.1 \% \mathrm{HER} 2+$ and $26 \%$ TNBC (20). Thus, Indian women have a higher proportion of TNBC, whereas TNBCs are less prevalent in European (around 9\%) and non-African American females (16\%) $(21,22)$. This is consistent with a study published in 2014 comparing the incidence of breast cancer subtypes among Indian, Hispanic, African-American, Chinese and Non-Hispanic women and it showed that Indian women had a higher incidence of TNBCs than any other race, and it was significantly higher in younger women (23). In this study, all cohorts were divided into two groups, namely a younger group (age <40) and an older group (age $>40$ ). The early onset of breast cancer was studied in the younger group. In this group, stage 2 and stage 3 cancers were observed to be highest in the Indian cohort $(88 \%)$, followed by African American (66\%), Chinese (62\%), Hispanic (60\%), and Non-Hispanic women (36\%).

\section{TNBCs and HER2+ Cases Showed the Highest Immune Response}

TIICs were counted and categorized by a pathologist. Greater than $10 \%$ of stromal TIICs was considered to represent immune responsiveness. Figure $1 \mathrm{~b}$ and Table 2 show the distribution of cases based on TIICs and breast cancer subtypes. Representative images showing varying densities of TIICs are seen in Figures 1c-f. 85/132 (64.4\%) TNBC cases had more than 10\% TIICs, followed by $49 / 77$ (63.6\%) HER2+ cases and 76/183 (41.5\%) HR+ cases (Table 2). TNBCs and HER2+ cases showed the highest immune response.

\section{PD-1 Staining in TIICs}

IHC was performed to identify the number of PD-1 positive TIICs within various breast cancer subtypes. The overall staining of tumorinfiltrating immune cells for PD-1 is shown in Table 3 and Figures $2 \mathrm{a}$ and $2 \mathrm{~b}$. Since seven sections were lost, 385 sections were reviewed. Around $14 \%$ of all cases (55/385) showed TIICs stained positively for PD-1 (Table 3), with the positivity rate in the subgroups being: TNBC 
21/55 (38.2\%); HER2 17/55 (31\%); and HR+ 17/55 (30\%). The majority $(83.6 \%)$ of cases across the breast cancer subtypes $(46 / 55)$ showed staining in $\leq 10 \%$ TIICs. None of the cases showed PD-1 staining in $>50 \%$ of TIICs. When taken together, the relatively "bad" prognostic groups of TNBC and HER2 positive accounted for $69.1 \%$ of cases with PD-1 stained TIICs.

\section{TNBCs Show Higher PD-L1 Positive Tumor Cells}

IHC was performed on breast cancer sections to detect PD-L1 positivity in the tumor. Since seven sections were lost and seven more were not suitable for assessment in the tumor area, 378 sections were reviewed. Overall, across all breast cancer subtypes, 41 out of 378 samples $(-11 \%)$ stained positively for PD-L1 in tumor cells (Figure 3a). IHC showed membranous staining of PD-L1 on tumor cells and varying degrees of staining were observed where some showed partial staining of the cell membrane and others showed complete staining (Figures 3C-F). The results of the overall staining of tumor cells for PD-L1 are shown in Table 4. Of the 41 cases which stained positive for PD-L1, 15 cases $(36.6 \%)$ showed PD-L1 in more than $10 \%$ of cells. Seventeen out of 41 cases that were positive for PD-L1 were TNBCs
(41.4\%). When taken together, the relatively "bad" prognostic groups, TNBC and HER2+, accounted for (26/41) 63.4\% of cases showing PD-L1 positive tumor cells. Amongst all TNBC cases, $12.9 \%$ (17 out of 132) of cases were PD-L1 positive (Table 4).

\section{TNBCs show higher PD-L1 expressing TIICs}

In the IHC performed for PD-L1 above, the PD-L1 expression in TIICs was assessed. As seven sections were lost, 385 sections were reviewed. Overall, across all breast cancer subtypes, 151/385 (39.2\%) cases showed positive staining for PD-L1 in TIICs (Figure 4a). 59/151 (39.0\%) positive cases showed PD-L1 staining of $>10 \%$ and $92 / 151$ $(60.9 \%)$ positive cases showed staining in $\leq 10 \%$ cells. $68 / 151(45.0 \%)$ positive cases were TNBC (Figure 4b; Table 5). In absolute numbers, 68/132 (51.5\%) of TNBC cases exhibited PD-L1 positive TIICs. When taken together, the relatively "bad" prognostic groups of TNBC and HER 2 + accounted for $67.6 \%$ of all cases with PD-L1 stained TIICs.

The first study on PD-L1 expression in breast cancer, comprising 44 patients from Saudi Arabia, was published in 2006. In this study, 15 of the $44(34 \%)$ cases had PD-L1 expression in tumor cells and 18 of 44

Table 1. Distribution of estrogen receptor (ER), progesterone receptor (PR) and HER2 in the 392 cases analyzed

\begin{tabular}{|lcccc} 
& $\begin{array}{c}\text { Hormone receptor + (HER-2 } \\
\text { +/-): a surrogate for luminal A } \\
\text { \& B subtypes (HR+) }\end{array}$ & $\begin{array}{c}\text { HER-2 positive, HR-ve: } \\
\text { a surrogate for HER-2 } \\
\text { overexpressing subtype (HER-2+) }\end{array}$ & $\begin{array}{c}\text { TNBC: a surrogate for basal- } \\
\text { like subtypes (ER/PR/HER-2 } \\
\text {-ve) }\end{array}$ & $\begin{array}{c}\text { Total } \\
\text { Number of cases }\end{array}$ \\
\hline Percentage & 183 & 77 & 132 & 392 \\
\hline
\end{tabular}

TNBC: Triple-negative breast cancer, HER2: Human epidermal growth factor receptor 2, HR+: Hormone receptor positivity

Table 2. Distribution of tumor-infiltrating immune cells (TIICS) in the three major breast cancer subtypes

\begin{tabular}{|lccc}
\hline TIICs $\%$ & HR+, HER2+/- & HER2+, HR- & TNBC \\
\hline $\mathbf{0} \%$ & 26 & 03 & 05 \\
$\mathbf{1} \%-\mathbf{1 0} \%$ & 81 & 25 & 42 \\
$\mathbf{1 1 \% - 5 0 \%}$ & 74 & 44 & 69 \\
$\mathbf{>} \mathbf{5 0} \%$ & 02 & 77 & 16 \\
Total & 183 & & 132 \\
\hline P-value <0.0001, chi-squared test, chi-square value = 35.96, degrees of freedom = 6. & \\
HER2: Human epidermal growth factor receptor 2, TNBC: Triple-negative breast cancer, HR+: Hormone receptor positivity &
\end{tabular}

Table 3. Percentage of tumor-infiltrating immune cells (TIICS) staining for PD-1

\begin{tabular}{|c|c|c|c|}
\hline Percentage of TIICs sta & HR+, HER2+/- & HER2+ HR- & TNBC \\
\hline $1 \%-4 \%$ & 6 & 5 & 10 \\
\hline $5 \%-10 \%$ & 11 & 9 & 5 \\
\hline $11 \%-50 \%$ & 0 & 3 & 6 \\
\hline$>50$ & 0 & 0 & 0 \\
\hline Total $=55 / 385(14.3 \%)$ & $17 / 55(30.9 \%)$ & $17 / 55(30.9 \%)$ & $21 / 55(38.18 \%)$ \\
\hline
\end{tabular}

p-value $>0.05$, chi-squared tests are not valid for contingency tables with values of 0 , hence $5 \%-10 \%, 11 \%-50 \%$ and $>50 \%$ were merged for statistical analysis, chi-squared value $=1.406$, degrees of freedom $=2$.

HER2: Human epidermal growth factor receptor 2, TNBC: Triple-negative breast cancer, HR+: Hormone receptor positivity 
(41\%) had tumor-infiltrating lymphocytes (TILs) expressing PD-L1 (24). A study with 650 cases from Switzerland had shown a higher PDL1 expression in tumor cells ( $23.4 \%$ of the cases) and a small subset of cases (9.2\%) showed PD-L1 positive TILs (14). A large retrospective study involving 5,763 patients from METABRIC genomic study, SEARCH observational study and NEAT randomized controlled trial from the United Kingdom showed that TIICs expressed PD-L1 in only $6 \%$ of the cases and by tumor cells in $1.7 \%$ cases. $19 \%$ of TNBCs had PD-L1 positive immune cells in their study (25). In contrast, our study showed $11 \%$ of the cases expressing PD-L1 in tumor cells and $39 \%$ of the cases expressing PD-L1 in TIICs, which is relatively higher. We also observed as high as $51.5 \%$ of TNBCs with PD-L1 positive TIICs.

\section{Discussion and Conclusion}

\section{RT-qPCR for Detecting PD-L1 Expression}

We undertook a pilot study to assess if qPCR can be used to detect PD-L1 transcript expression. Fresh tissue samples (76) were collected and quantitative PCR was conducted successfully for 29 samples. The PD-L1 expression levels determined by RT-qPCR were scored based

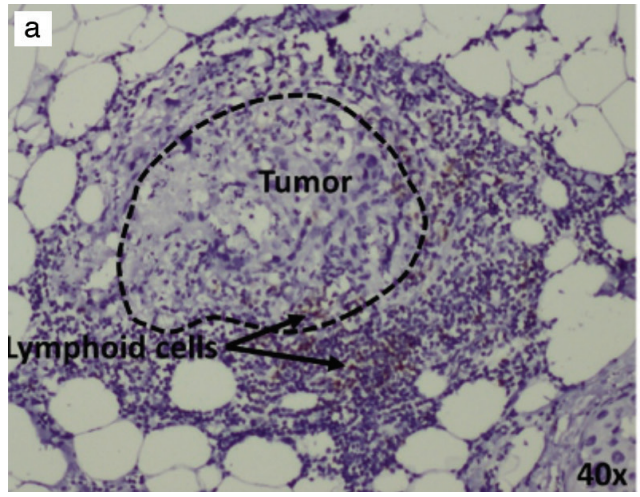

on $\Delta$ Ct values. RT-qPCR could detect PD-L1 transcripts in all 29 samples. The data showed that PD-L1 has a heterogeneous expression (Figure 5a). Fifteen samples showed lower expression relative to the housekeeping gene HPRT, while 14 samples showed higher expression than HPRT. Of the 29 tumor samples, for nine samples, we additionally procured adjacent normal samples. When compared to adjacent normal tissues, 6/9 tumors had higher expression of PDL1 and 3/9 had lower expression of PD-L1 compared to respective adjacent normal tissue (Figure 5b). Thus, RT-qPCR could detect the mRNA of PD-L1. However, use of qPCR for diagnostic purposes should be assessed further in experiments with larger sample size and should also be correlated with the pathologist's IHC scoring.

\section{Limitations of the Study}

Research-use antibodies were used instead of IVD clones, as the latter are expensive and were not supported by the funding agency. Further, the intention of this study was to check only the expression of PDL1 protein and not for any therapeutic intervention. Comparison of PCR with IHC could not be made because of the limited number of good quality RNA samples from resected tumors owing to technical/ procedural issues.

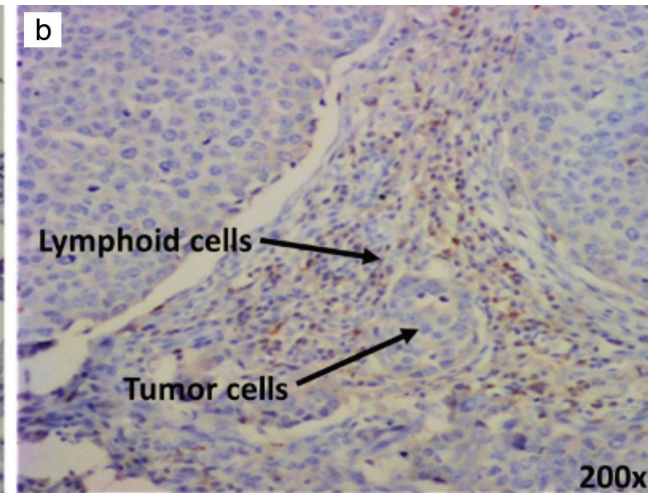

Figure 2. a) Lymphoid cells with PD-1 around a focus of tumor cells. b) PD-1 positive immune cells around unstained tumor cells PD-1: Programmed cell death protein 1

Table 4. Percentage of tumor cells showing PD-L1 positivity in breast cancer subtypes

\begin{tabular}{|lccc}
\hline Percentage of tumour cells stained/100 viable tumour cells & HR+, HER2+/- & HER2+, HR- & TNBC \\
\hline 1\%-9\% & 9 & 2 & 7 \\
$10 \%$ and above & 6 & 2 & 7 \\
Total = 41/378 (10.85\%) & $15 / 41(36.6 \%)$ & $9 / 41(21.9 \%)$ & $17 / 41(41.46 \%)$
\end{tabular}

p-value $>0.05$, chi-squared test, chi-square value $=1.03$, degrees of freedom $=2$.

HER2: Human epidermal growth factor receptor 2, TNBC: Triple-negative breast cancer, HR+: Hormone receptor positivity

Table 5. Staining of TIICs for PD-L1

\begin{tabular}{|lccc}
\hline Percentage of TIICs stained/100 viable tumor cells & HR+, HER-2+/- & HER-2+, HR- & TNBC \\
\hline 1\%-10\% & 34 & 24 & 34 \\
$>10 \%$ & 15 & 10 & 34 \\
Total = 151/385 (39.2\%) & $49 / 151(32.5 \%)$ & $34 / 151(22.5 \%)$ & $68 / 151(45 \%)$
\end{tabular}

p-value $>0.05$, statistically not significant, chi-squared tests are not valid for contingency tables with values of 0 , hence $11 \%-50 \%$ and $>50 \%$ were merged for statistical analysis, chi-squared value $=7.475$, degrees of freedom $=4$. 


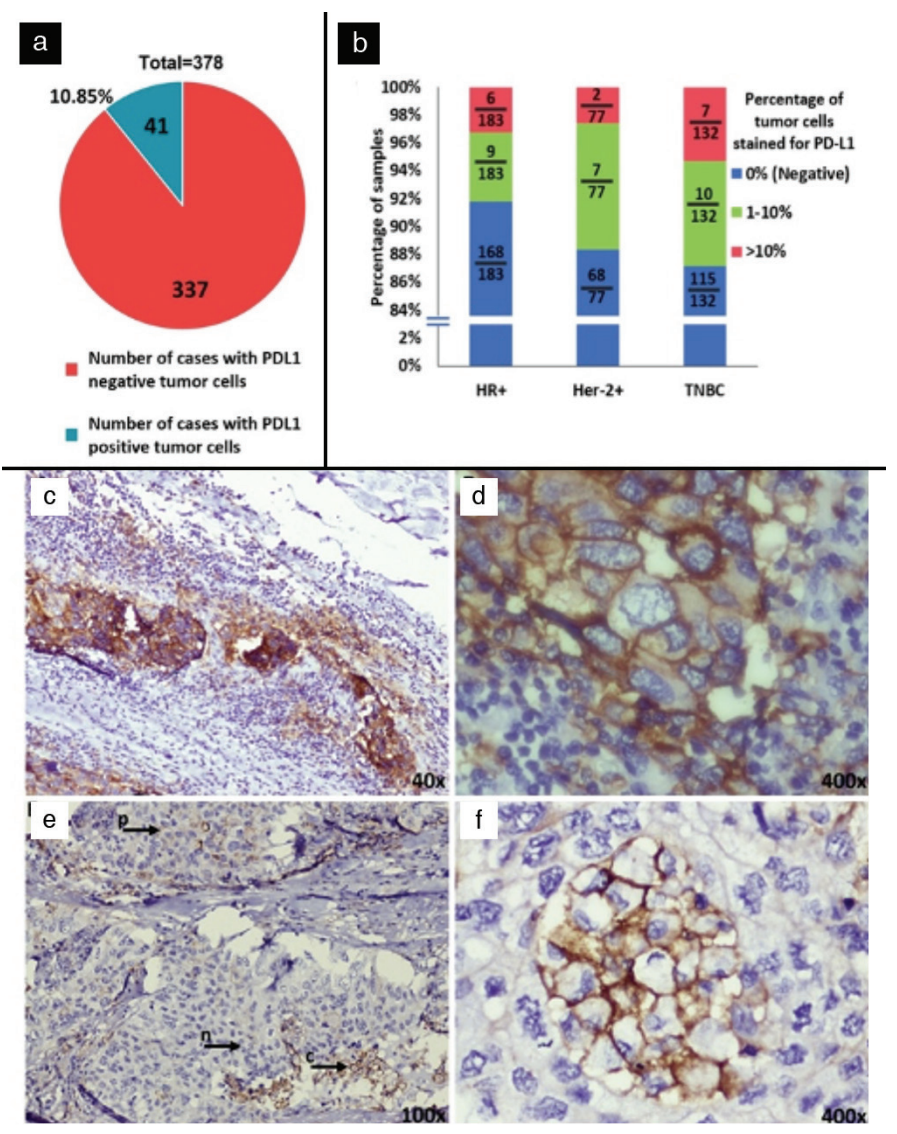

Figure 3. a) Pie chart representing breast cancer cases ( $n=378$, after eliminating 14 cases in which IHC failed) with tumor cells expressing PD-L1 (41/378). b) Stacked column graph depicting he percentage of cases expressing PD-L1 on tumor cells. Representative image of immunohistochemistry (IHC) of PD-L1 c) with dense staining on tumor cells. d) with strong membranous staining of PD-L1 e) arrows indicate tumor cells with nil ( $\mathrm{n})$, partial ( $\mathrm{p}$ ) and complete (c) membranous staining in the same field of view. f) PD-L1 expression observed in tumor cells and not the adjacent lymphoid cells.

PD-L1: Programmed cell death ligand 1

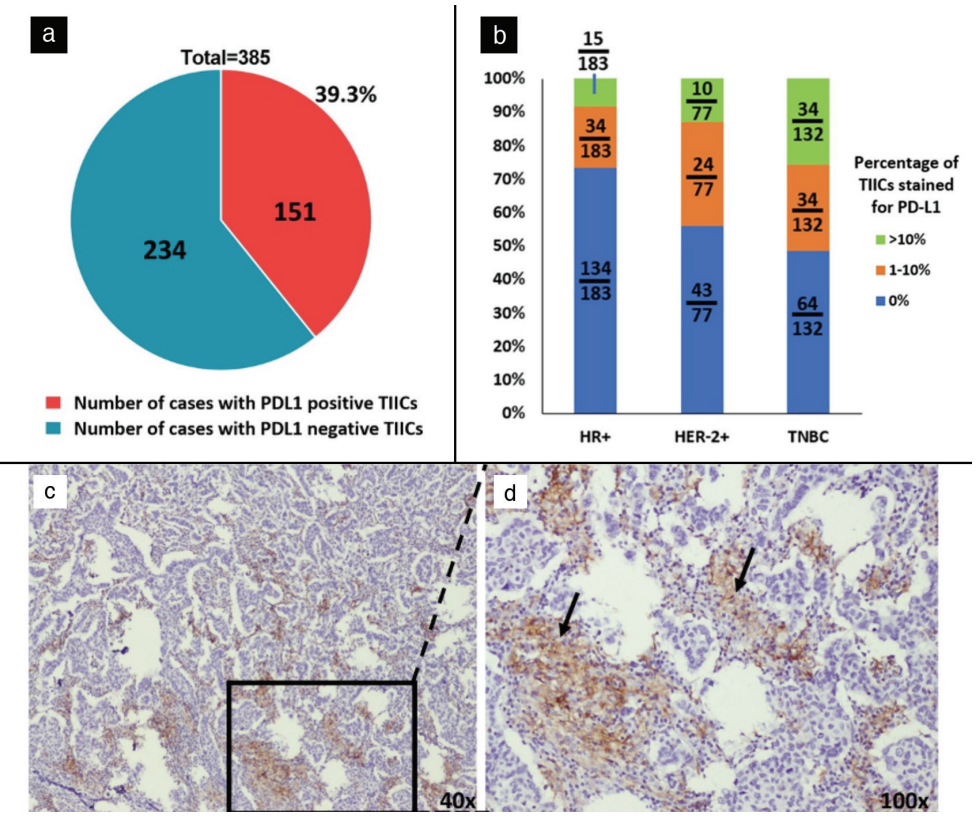

Figure 4. a) Pie chart representing breast cancer cases ( $n=385$, after eliminating seven cases in which IHC failed) which had tumor infiltrating immune cells (TIICS) expressing PD-L1 (151/385). b) Column graph depicting the distribution of cases with TIICs stained for PD-L1 (categorized by breast cancer subtypes). c) Lymphoid cells with PD-L1 around tumor cells. d) Magnified image of c showing the infiltration of PD-L1 positive immune cells. 

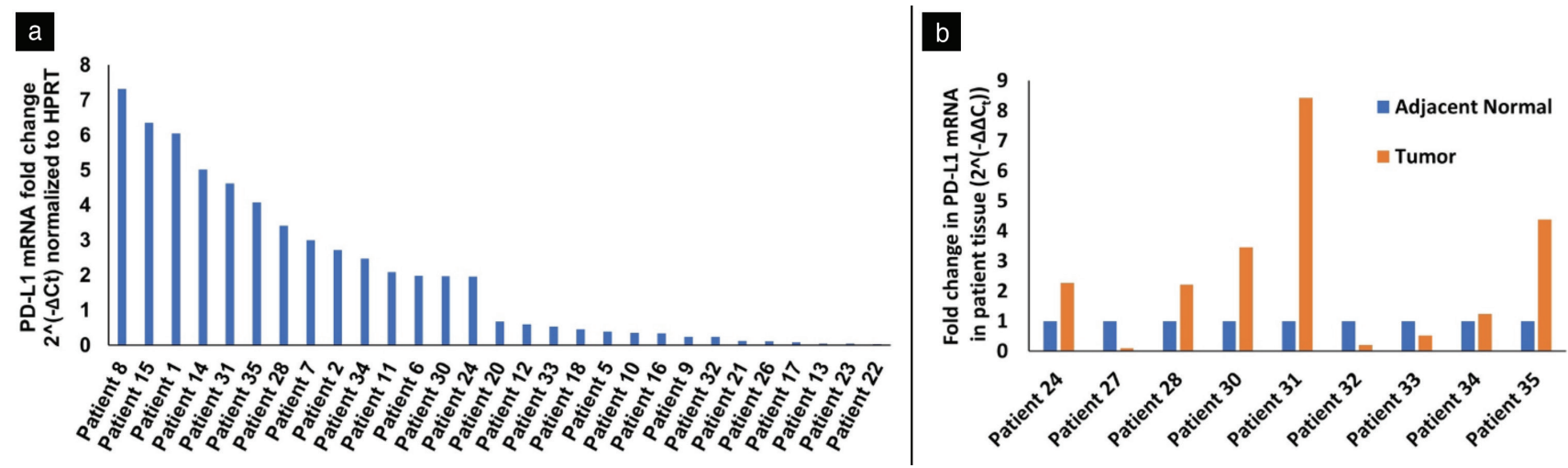

Figure 5. a) PD-L1 mRNA expression normalized to HPRT in 33 tumor tissue samples. Seventeen of the samples showed PD-L1 mRNA levels lower than HPRT while 16 of them showed higher than HPRT b) PD-L1 mRNA expression in tumor and adjacent normal tissue from nine patients.

HPRT: Hypoxanthine-guanine phosphoribosyl transferase, PD-L1: Programmed cell death ligand 1

In conclusion, we had quantified the PD-L1 levels in breast cancer for the first time in an Indian cohort. Around $92 \%$ of cases had TIICs and about $39 \%$ of cases showed PD-L1 staining in TIICs. Further, 52\% (68/132) of TNBC cases had PD-L1-expressing TIICs. Although breast cancers are immunogenic, this immune response may be suppressed by the PD-L1 expressing TIICs. This has opened an opportunity to explore anti-PD-L1 therapy to treat the most aggressive TNBCs in the Indian population.

PD-L1 is regulated mainly by interferon I and II pathways. Interferons signal through multiple pathways via JAK-STAT transcription factors to up-regulate the expression of PD-L1. Signals from the ERK pathway converge on STAT1, while signals from PI3K/Akt pathway converge on STAT3 and induce PD-L1 expression (26). Recent studies have shown AMP-activated protein kinase (AMPK) to phosphorylate PD-L1 at S195, which leads to abnormal glycosylation leading to degradation of PD-L1 (27). Interestingly, we observed that AMPK inhibition with pharmacological inhibitor Compound $\mathrm{C}$ led to an increase in PD-L1 expression in the MDA-MB-231 (TNBC) cell line while reduced PD-L1 expression in MCF7 ( $\mathrm{HR}+$ ) (Supplementary Figure 1). AMPK activators, such as the anti-diabetic drug metformin, and inhibitors can be used as an immunomodulator. Our lab is currently investigating the role of AMPK in the regulation of PD-L1 in various stages of cancer. Understanding the molecular mechanisms involved in the PD-1/PD-L1 axis is likely to unveil other pharmacological targets in the future.

Acknowledgement: We thank Rajiv Gandhi University of Health Sciences, Bengaluru, India for financial support, Rachana, Prashanth Kumar and Narayanaswamy for technical support during sample collection and immunohistochemistry.

Ethics Committee Approval: This study was approved by the Medical Ethics Committee (No: MEC/001, date: April 30, 2016).

Informed Consent: It was obtained.

Peer-review: Externally peer-reviewed.

\section{Authorship Contributions}

Conception: A.R., R.V.K.; Design: A.R., R.V.K.; Supervision: S.M.N., S.M.C.B., A.R., R.V.K.; Data Analysis and/or Interpretation: K.R.B., R.V.K.;
Manuscript Review: S.M.N., R.C., S.M.C.B., A.R., R.V.K.; Writing: K.R.B., K.D., S.M.N., R.C., S.M.C.B., A.R., R.V.K.

Conflict of Interest: No conflict of interest declared by the authors.

Financial Disclosure: This study was funded by Rajiv Gandhi University of Health Sciences, Bengaluru, India (ID: RI003, 2016-2018).

\section{References}

1. Bray F, Ferlay J, Soerjomataram I, Siegel RL, Torre LA, Jemal A. Global cancer statistics 2018: GLOBOCAN estimates of incidence and mortality worldwide for 36 cancers in 185 countries. CA Cancer J Clin 2018; 68: 394-424. (PMID: 30207593) [Crossref]

2. Eroles P, Bosch A, Alejandro Pérez-Fidalgo J, Lluch A. Molecular biology in breast cancer: Intrinsic subtypes and signaling pathways. Cancer Treat Rev 2012;38:698-707. (PMID: 22178455) [Crossref]

3. Vasconcelos I, Hussainzada A, Berger S, Fietze E, Linke J, Siedentopf F, et al. The St. Gallen surrogate classification for breast cancer subtypes successfully predicts tumor presenting features, nodal involvement, recurrence patterns and disease-free survival. Breast 2016; 29: 181-185. (PMID: 27544822) [Crossref]

4. Schreiber RD, Old LJ, Smyth MJ. Cancer Immunoediting: Integrating immunity's roles in cancer suppression and promotion. Science 2011; 331: 1565-1570. (PMID: 21436444) [Crossref]

5. Dong H, Strome SE, Salomao DR, Tamura H, Hirano F, Flies DB, et al. Tumor-associated B7-H1 promotes T-cell apoptosis: A potential mechanism of immune evasion. Nat Med 2002;8:793-800. (PMID: 12091876) [Crossref]

6. Tseng S-Y, Otsuji M, Gorski K, Huang X, Slansky JE, Pai SI, et al. B7-Dc, a new dendritic cell molecule with potent costimulatory properties for T cell. J Exp Med 2001;193:839-46. PMID: 11283156) [Crossref]

7. Francisco LM, Salinas VH, Brown KE, Vanguri VK, Freeman GJ, Kuchroo VK, et al. PD-L1 regulates the development, maintenance, and function of induced regulatory T cells. J Exp Med 2009; 206: 3015-3029. (PMID: 20008522) [Crossref]

8. Zou W, Chen L. Inhibitory B7-family molecules in the tumour microenvironment. Nat Rev Immunol 2008; 8: 467-477. (PMID: 18500231) [Crossref]

9. Iwai Y, Ishida M, Tanaka Y, Okazaki T, Honjo T, Minato N. Involvement of PD-L1 on tumor cells in the escape from host immune system and tumor immunotherapy by PD-L1 blockade. Proc Natl Acad Sci U S A 2002; 99: 12293-12297. (PMID: 12218188) [Crossref] 
10. Gong J, Chehrazi-Raffle A, Reddi S, Salgia R. Development of PD-1 and PD-L1 inhibitors as a form of cancer immunotherapy: a comprehensive review of registration trials and future considerations. J Immunother Cancer 2018;6:8. (PMID: 29357948) [Crossref]

11. Krishnamurthy S, Poornima R, Challa VR, Goud YGB. Triple negative breast cancer - Our experience and review. Indian J Surg Oncol 2012; 3: 12-16. (PMID: 23449631) [Crossref]

12. Bayraktar $S$, Batoo $S$, Okuno $S$, Glück $S$. Immunotherapy in breast cancer. J Carcinog 2019; 18: 2. (PMID: 31160888) [Crossref]

13. Schmid P, Rugo HS, Adams S, Schneeweiss A, Barrios $\mathrm{CH}$, Iwata $\mathrm{H}$, et al. Atezolizumab plus nab-paclitaxel as first-line treatment for unresectable, locally advanced or metastatic triple-negative breast cancer (IMpassion130): updated efficacy results from a randomised, doubleblind, placebo-controlled, phase 3 trial. Lancet Oncol 2020; 21: 44-59. (PMID: 31786121) [Crossref]

14. Muenst S, Schaerli AR, Gao F, Däster S, Trella E, Droeser RA, et al. Expression of programmed death ligand 1 (PD-L1) is associated with poor prognosis in human breast cancer. Breast Cancer Res Treat 2014; 146: 15-24. (PMID: 24842267) [Crossref]

15. Sabatier R, Finetti P, Mamessier E, Adelaide J, Chaffanet M, Ali HR, et al. Prognostic and predictive value of PDL1 expression in breast cancer. Oncotarget 2015; 6: 5449-5464. (PMID: 25669979) [Crossref]

16. Boffetta P, Hainaut P, editors. Encyclopedia of cancer. 3rd ed. Massachusetts: Academic Press; 2019.

17. Gonzalez-Ericsson PI, Stovgaard ES, Sua LF, Reisenbichler E, Kos Z, Carter JM, et al. The path to a better biomarker: application of a risk management framework for the implementation of PD-L1 and TILs as Immuno-oncology biomarkers in breast cancer clinical trials and daily practice. J Pathol 2020; 250: 667-684. (PMID: 32129476) [Crossref]

18. Salgado R, Denkert C, Demaria S, Sirtaine N, Klauschen F, Pruneri G, et al. The evaluation of tumor-infiltrating lymphocytes (TILs) in breast cancer: recommendations by an international TILs working group 2014 . Ann Oncol 2015; 26: 259-271. (PMID: 25214542) [Crossref]

19. Kumar RV, Panwar D, Amirtham U, Premalata CS, Gopal C, Narayana $\mathrm{SM}$, et al. Estrogen receptor, progesterone receptor, and human epidermal growth factor receptor-2 status in breast cancer: a retrospective study of 5436 women from a regional cancer center in South India. South Asian J Cancer 2018;7:7-10. (PMID: 25214542) [Crossref]

20. Pandit P, Patil R, Palwe V, Gandhe S, Patil R, Nagarkar R. Prevalence of molecular subtypes of breast cancer: a single institutional experience of 2062 patients. Eur J Breast Health 2019; 16: 39-43. (PMID: 31912012) [Crossref]

21. Carey LA, Perou CM, Livasy CA, Dressler LG, Cowan D, Conway K, et al. Race, breast cancer subtypes, and survival in the Carolina Breast Cancer Study. JAMA 2006; 295: 2492-2502. (PMID: 16757721) [Crossref]

22. Kondov B, Milenkovikj Z, Kondov G, Petrushevska G, Basheska N, Bogdanovska-Todorovska $M$, et al. Presentation of the molecular subtypes of breast cancer detected by immunohistochemistry in surgically treated patients. Open Access Maced J Med Sci 2018; 6: 961-967. (PMID: 29983785) [Crossref]

23. Singh M, Ding Y, Zhang L-Y, Song D, Gong Y, Adams S, et al. Distinct breast cancer subtypes in women with early-onset disease across races. Am J Cancer Res 2014; 4: 337-352. (PMID: 25057437) [Crossref]

24. Ghebeh H, Mohammed S, Al-Omair A, Qattan A, Lehe C, Al-Qudaihi G, et al. The B7-H1 (PD-L1) T lymphocyte-inhibitory molecule is expressed in breast cancer patients with infiltrating ductal carcinoma: correlation with important high-risk prognostic factors. Neoplasia 2006; 8: 190-198. (PMID: 16611412) [Crossref]

25. Ali HR, Glont SE, Blows FM, Provenzano E, Dawson SJ, Liu B, et al. PD-L1 protein expression in breast cancer is rare, enriched in basal-like tumors and associated with infiltrating lymphocytes. Ann Oncol 2015; 26: 1488-1493. (PMID: 25897014) [Crossref]

26. Vranic S, Cyprian FS, Gatalica Z, Palazzo J. PD-L1 status in breast cancer: current view and perspectives. Semin Cancer Biol 2019; 72: 146154. (PMID: 31883913) [Crossref]

27. Cha JH, Yang WH, Xia W, Wei Y, Chan LC, Lim SO, et al. Metformin promotes anti-tumor immunity via endoplasmic-reticulum-associated degradation of PD-L1. Mol Cell 2018; 71: 606-620.e7. (PMID: 30118680) [Crossref]

\section{Supplementary data}
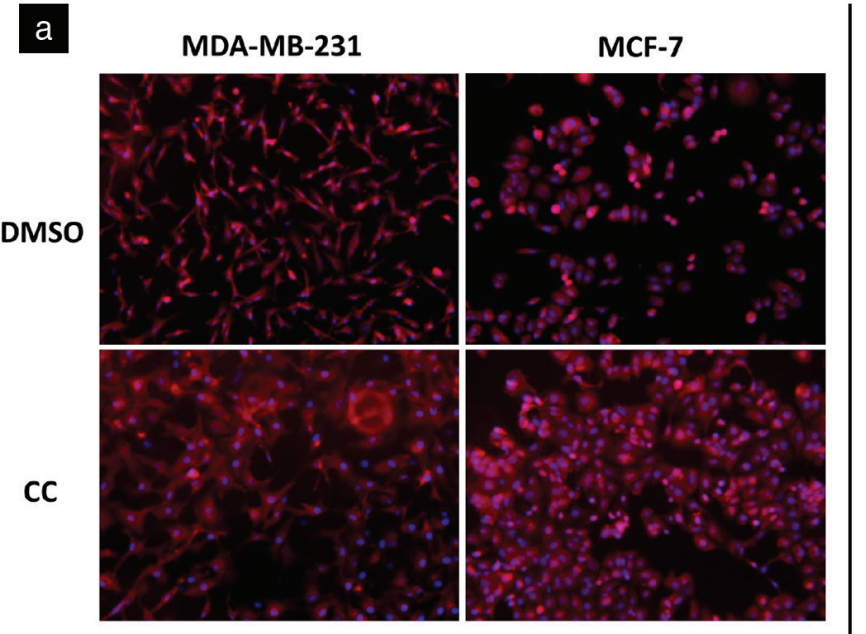

b

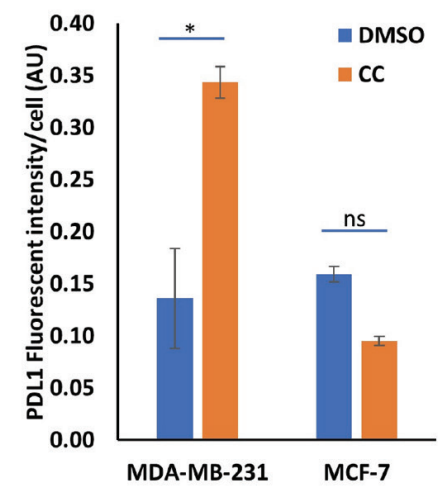

Supplementary Figure 1. a) Immunocytochemistry showing PD-L1 expression in MDA-MB-231 and MCF7 cell lines. DMSO - vehicle control, CC - Compound C - a pharmacological inhibitor of AMPK. b) Quantification of PD-L1 fluorescence intensity in immunocytochemistry. MDAMB-231 showed increased PD-L1 expression on CC treatment while MCF7 showed reduced PD-L1 expression on CC treatment, AU - arbitrary units, mean fluorescence intensity was calculated from at least 100 cells, $n=4$, * $\mathrm{p}$-value $<0.05$

PD-1: Programmed cell death protein 1, DMSO: Dimethylsulfoxide, CC: Compound, ns: Not significant 